\title{
Conservative Quantum Computing
}

\author{
Masanao Ozawa \\ Graduate School of Information Sciences, Tôhoku University, Aoba-ku, Sendai 980-8579, Japan \\ Center for Photonic Communication and Computing, Department of Electrical and Computer Engineering, Northwestern \\ University, Evanston, IL 60208
}

\begin{abstract}
Conservation laws limit the accuracy of physical implementations of elementary quantum logic gates. If the computational basis is represented by a component of spin and physical implementations obey the angular momentum conservation law, any physically realizable unitary operators with size less than $n$ qubits cannot implement the controlled-NOT gate within the error probability $1 /\left(4 n^{2}\right)$, where the size is defined as the total number of the computational qubits and the ancilla qubits. An analogous limit for bosonic ancillae is also obtained to show that the lower bound of the error probability is inversely proportional to the average number of photons. Any set of universal gates inevitably obeys a related limitation with error probability $O\left(1 / n^{2}\right)$. To circumvent the above or related limitations yielded by conservation laws, it is recommended that the computational basis should be chosen as the one commuting with the additively conserved quantities.
\end{abstract}

PACS numbers: 03.67.Lx, 03.67.-a, 03.65.Ta

Since the discovery of Shor's algorithm [1], physical realization of quantum computers is one of the major topics in physics. One of the formidable obstacles to the realization of quantum computers is the decoherence induced by the environment. The theory of quantum error correction and the theory of fault-tolerant quantum computing have been developed to overcome this difficulty [2.,3]. One of the main achievements of this field is the threshold theorem: Provided the noise in individual quantum gates is below a certain threshold it is possible to efficiently perform an arbitrarily large quantum computing. However, the threshold is rather demanding and the problem turns to whether there is any fundamental limit for implementing quantum gates. Recently, Lloyd [4] and Ng [5] have discussed how fundamental constants provide limits on speed and memory of quantum computers. Here, I will propose another approach based on conservation laws.

If we consider the ultimate performance of computing allowed by the laws of physics, elementary quantum gates should be isolated and small, so that the corresponding unitary operators should satisfy fundamental symmetries, or conservation laws. From this point of view, it is likely that the degree of conflict with a conservation law depends on the nature of its logic to be performed and that the imperfection can be reduced by increasing the size of implementation. However, no serious investigation has ever taken place. In this letter we model qubits as spin $1 / 2$ objects and investigate the quantum limit induced by the angular momentum conservation law. We show that although the SWAP gate has no conflict with the conservation law, the controlledNOT gate, which is one of the universal quantum logic gates, cannot be implemented by any 2 -qubit rotationally invariant unitary operation within error probability $1 / 16$. Thus, to obtain more accuracy, we need to blow up the unitary operation to an ancilla system. Then, the size of an implementation of the quantum gate is defined as the total number of qubits in the computational basis and the ancilla. It is shown that any physically realizable unitary operator with size less than $n$ qubits cannot implement the controlled-NOT gate within the error probability $1 /\left(4 n^{2}\right)$. An analogous limit for bosonic ancillae will be also obtained by defining the size of the ancilla as 2 times the square root of the average number of photons, and thus the lower bound is inversely proportional to the average number of photons. It is also shown that in any set of universal gates, for any size limit $s$ there is at least one gate which cannot be implemented within the error probability $1 /\left(k s^{2}\right)$ for some constant $k$. Thus, we cannot circumvent this limitation by a clever choice of the set of universal gates.

Let $U_{\mathrm{CN}}$ be a controlled-NOT gate on a 2-qubit system $\mathbf{C}+\mathbf{T}$. Let $X_{i}, Y_{i}$, and $Z_{i}$ be the Pauli operators of qubit $\mathbf{C}$ for $i=1$ or qubit $\mathbf{T}$ for $i=2$ defined by $X_{i}=$ $|1\rangle\langle 0|+| 0\rangle\left\langle 1\left|, Y_{i}=i\right| 1\right\rangle\langle 0|-i| 0\rangle\langle 1|$, and $Z_{i}=|0\rangle\langle 0|-$ $|1\rangle\langle 1|$ with the computational basis $\{|0\rangle,|1\rangle\}$. On the computational basis, $U_{\mathrm{CN}}$ acts as $U_{\mathrm{CN}}|a, b\rangle=|a, b \oplus a\rangle$ for $a, b=0,1$, where $\oplus$ denotes the addition modulo 2 . Thus, in particular, we have

$$
U_{\mathrm{CN}}|a, 0\rangle=|a, a\rangle
$$

for $a=0,1$. The above relation shows that the unitary operator $U_{\mathrm{CN}}$ serves as an interaction between the "object" $\mathbf{C}$ and the "probe" $\mathbf{T}$ for a measurement of $Z_{1}$ satisfying the projection postulate. Thus, by the WignerAraki-Yanase theorem [6.7], if there are additive conserved quantities not commuting with $Z_{1}$, the unitary operator $U_{\mathrm{CN}}$ cannot be implemented. To be precise, let $L_{1}$ and $L_{2}$ be a pair of observables of $\mathbf{C}$ and $\mathbf{T}$, respectively, such that

$$
\left[Z_{1}, L_{1}\right] \neq 0 .
$$

Then, the controlled-NOT gate $U_{\mathrm{CN}}$ cannot satisfy the conservation law [8]

$$
\left[U_{\mathrm{CN}}, L_{1}+L_{2}\right]=0 .
$$


A simple proof runs as follows. Assume Eq. (3). If $a \neq b$, we have

$$
\begin{aligned}
\left\langle a\left|L_{1}\right| b\right\rangle & =\left\langle a, 0\left|L_{1}+L_{2}\right| b, 0\right\rangle \\
& =\left\langle a, 0\left|U_{\mathrm{CN}}^{\dagger}\left(L_{1}+L_{2}\right) U_{\mathrm{CN}}\right| b, 0\right\rangle \\
& =\left\langle a, a\left|L_{1}+L_{2}\right| b, b\right\rangle=0 .
\end{aligned}
$$

Thus, $L_{1}$ is diagonal in the computational basis of $\mathbf{C}$. Therefore, if $L_{1}$ does not commute with $Z_{1}$, then $U_{\mathrm{CN}}$ cannot satisfy the conservation law (3). In particular, $U_{\mathrm{CN}}$ cannot be implemented in the presence of the angular momentum conservation law.

The above impossibility of implementation depends on the logic. Despite the limitation on the controlled-NOT gate, the SWAP gate $U_{\mathrm{SWAP}}$, defined by $U_{\mathrm{SWAP}}|a, b\rangle=$ $|b, a\rangle$ for $a, b=0,1$, can be implemented precisely under the angular momentum conservation law. In fact, the SWAP gate can be precisely implemented as [9]

$$
U_{\mathrm{SWAP}}=\exp \frac{-i \pi}{4}\left(-1+X_{1} X_{2}+Y_{1} Y_{2}+Z_{1} Z_{2}\right) .
$$

In order to construct a physical implementation of $U_{\mathrm{CN}}$, the above consideration suggests the need for blowing up the unitary operation to a larger system including additional qubits. Let $\alpha=(U,|\xi\rangle)$ be a physical implementation of $U_{\mathrm{CN}}$ defined by a unitary operator $U$ on the system $\mathbf{C}+\mathbf{T}+\mathbf{A}$, where $\mathbf{A}$ is a quantum system called the ancilla, and a state vector $|\xi\rangle$ of the ancilla, in which the ancilla is prepared at the time at which $U$ is turned on. The implementation $\alpha=(U,|\xi\rangle)$ defines a trace-preserving quantum operation $\mathcal{E}_{\alpha}$ by

$$
\mathcal{E}_{\alpha}(\rho)=\operatorname{Tr}_{\mathbf{A}}\left[U(\rho \otimes|\xi\rangle\langle\xi|) U^{\dagger}\right]
$$

for any density operator $\rho$ of the system $\mathbf{C}+\mathbf{T}$, where $\operatorname{Tr}_{\mathbf{A}}$ stands for the partial trace over the system $\mathbf{A}$. On the other hand, the gate $U_{\mathrm{CN}}$ defines the trace-preserving quantum operation $\operatorname{ad} U_{\mathrm{CN}}$ by

$$
\operatorname{ad} U_{\mathrm{CN}}(\rho)=U_{\mathrm{CN}} \rho U_{\mathrm{CN}}^{\dagger}
$$

for any density operator $\rho$ of the system $\mathbf{C}+\mathbf{T}$.

How successful the implementation $(U,|\xi\rangle)$ has been is most appropriately measured by the completely bounded $(C B)$ distance 10] between two operations $\mathcal{E}_{\alpha}$ and $\operatorname{ad} U_{\mathrm{CN}}$ defined by

$$
D_{C B}\left(\mathcal{E}_{\alpha}, U_{\mathrm{CN}}\right)=\sup _{n, \rho} D\left(\mathcal{E}_{\alpha} \otimes \operatorname{id}_{n}(\rho), \operatorname{ad}_{\mathrm{CN}} \otimes \operatorname{id}_{n}(\rho)\right),
$$

where $n$ runs over positive integers, $\mathrm{id}_{n}$ is the identity operation on an $n$-level system $\mathbf{S}_{n}, \rho$ runs over density operators of the system $\mathbf{C}+\mathbf{T}+\mathbf{S}_{n}$, and $D\left(\sigma_{1}, \sigma_{2}\right)$ stands for the trace distance [2, p. 403] of two states $\sigma_{1}$ and $\sigma_{2}$. Since the trace distance of the above two states can be interpreted as an achievable upper bound on the so-called total variation distance of two probability distributions arising from measurements performed on the two output states of the corresponding gates [2, p. 405], we interpret $D_{C B}\left(\mathcal{E}_{\alpha}, U_{\mathrm{CN}}\right)$ as the worst error probability of operation $\mathcal{E}_{\alpha}$ in simulating the gate $U_{\mathrm{CN}}$ on any input state of any circuit including those two gates. We shall call $D_{C B}\left(\mathcal{E}_{\alpha}, U_{\mathrm{CN}}\right)$ the gate error probability of the implementation $\alpha$ of the gate $U_{\mathrm{CN}}$.

Another measure, which is more tractable in computations, is the gate fidelity [2, p. 418] defined by

$$
F\left(\mathcal{E}_{\alpha}, U_{\mathrm{CN}}\right)=\min _{|\psi\rangle} F(\psi)
$$

where $|\psi\rangle$ varies over all state vectors of $\mathbf{C}+\mathbf{T}$, and $F(\psi)$ is the fidelity of two states $U_{\mathrm{CN}}|\psi\rangle$ and $\mathcal{E}_{\alpha}(|\psi\rangle\langle\psi|)$ given by

$$
F(\psi)=\left\langle\psi\left|U_{\mathrm{CN}}^{\dagger} \mathcal{E}_{\alpha}(|\psi\rangle\langle\psi|) U_{\mathrm{CN}}\right| \psi\right\rangle^{1 / 2} .
$$

By the relation [2, p. 416]

$$
1-F\left(\mathcal{E}_{\alpha}, U_{\mathrm{CN}}\right)^{2} \leq D_{C B}\left(\mathcal{E}_{\alpha}, U_{\mathrm{CN}}\right),
$$

any lower bound of $1-F\left(\mathcal{E}_{\alpha}, U_{\mathrm{CN}}\right)^{2}$ gives a lower bound of the gate error probability. The operator $U$ and the operation $\mathcal{E}_{\alpha}$ is generally described by the following actions on computational basis states

$$
\begin{aligned}
U|a, b\rangle|\xi\rangle & =\sum_{c, d=0}^{1}|c, d\rangle\left|E_{c d}^{a b}\right\rangle \\
\mathcal{E}_{\alpha}(|a, b\rangle\langle a, b|) & =\sum_{i, j, k, l=0}^{1}|i, j\rangle\left\langle E_{k, l}^{a, b} \mid E_{i, j}^{a, b}\right\rangle\langle k, l|
\end{aligned}
$$

for $a, b=0,1$, where $\left|E_{c d}^{a b}\right\rangle$ is not necessarily normalized. It follows that the fidelity is given by

$$
F(a, b)=\|\left|E_{a, b \oplus a}^{a, b}\right\rangle \| .
$$

Now, we assume that there are additive conserved quantities $L_{1}, L_{2}$, and $L_{3}$ of systems $\mathbf{C}$, $\mathbf{T}$, and $\mathbf{A}$, respectively, so that the unitary operator $U$ should satisfy the conservation law

$$
\left[U, L_{1}+L_{2}+L_{3}\right]=0 .
$$

Since computational qubits, $\mathbf{C}$ and $\mathbf{T}$, should have the same physical structure, we naturally assume $\left\|L_{1}\right\|=$ $\left\|L_{2}\right\|$ for their operator norms.

Our problem is to find a good lower bound of the gate error probability (7) under the conservation law (14). In order to derive the lower bound from uncertainty relations, we introduce the deviation operators $D_{i j}$ of the system $\mathbf{C}+\mathbf{T}+\mathbf{A}$ for $i, j=1,2$ defined by

$$
D_{i j}=Z_{i}^{\prime}-Z_{j}
$$


where we write $A^{\prime}=U^{\dagger} A U$ for any operator $A$. The rootmean-square deviation $\delta_{i j}(\psi)$ on arbitrary input state $|\psi\rangle$ of $\mathbf{C}$ is defined as the root-mean-square of the deviation operator $D_{i j}$ in state $|\psi, 0, \xi\rangle=|\psi\rangle|0\rangle|\xi\rangle$, i.e.,

$$
\delta_{i j}(\psi)=\left\langle D_{i j}^{2}\right\rangle^{1 / 2}
$$

where $\langle\cdots\rangle$ abbreviates $\langle\psi, 0, \xi|\cdots| \psi, 0, \xi\rangle$. For any observable $A$, we shall denote by $\Delta A$ the standard deviation of $A$ defined by $\Delta A=\left\langle(A-\langle A\rangle)^{2}\right\rangle^{1 / 2}$. Then, we easily see

$$
\Delta D_{i j} \leq \delta_{i j}(\psi)
$$

for $i, j=1,2$. In the case where $U=U_{\mathrm{CN}}$, we have $D_{11}=$ $0, D_{12}=Z_{1}-Z_{2}, D_{21}=Z_{1}\left(Z_{2}-I\right)$, and $D_{22}=\left(Z_{1}-\right.$ I) $Z_{2}$, so that $\delta_{11}(\psi)=\delta_{21}(\psi)=0$ for any state $|\psi\rangle$ of C. Thus, the relation $\delta_{11}(\psi)^{2}+\delta_{21}(\psi)^{2}>0$ implies $U \neq$ $U_{\mathrm{CN}}$. Hence, the quantity $\delta_{11}(\psi)^{2}+\delta_{21}(\psi)^{2}$ measures a degree of imperfection.

Now, we shall evaluate $\delta_{11}(\psi)$ and $\delta_{21}(\psi)$ for a general implementation $\alpha=(U,|\xi\rangle)$ under the conservation law (14). From the conservation law (14) and the relations $\left[Z_{1}, L_{2}\right]=\left[Z_{1}, L_{3}\right]=0$, we have

$$
\left[Z_{1}, L_{1}\right]=\left[Z_{1}, L_{1}^{\prime}\right]+\left[Z_{1}, L_{2}^{\prime}\right]+\left[Z_{1}, L_{3}^{\prime}\right]
$$

From the definition of deviation operators, Eq. (15), we have

$$
\begin{gathered}
{\left[Z_{1}, L_{1}^{\prime}\right]=\left[L_{1}^{\prime}, D_{21}\right] \text { and }\left[Z_{1}, L_{2}^{\prime}\right]=\left[L_{2}^{\prime}, D_{11}\right]} \\
{\left[Z_{1}, L_{3}^{\prime}\right]=\left[L_{3}^{\prime}, D_{11}\right]=\left[L_{3}^{\prime}, D_{21}\right]}
\end{gathered}
$$

Thus, we have the following noise commutation relations

$$
\begin{aligned}
& {\left[Z_{1}, L_{1}\right]=\left[L_{1}^{\prime}, D_{21}\right]+\left[L_{2}^{\prime}, D_{11}\right]+\left[L_{3}^{\prime}, D_{11}\right],} \\
& {\left[Z_{1}, L_{1}\right]=\left[L_{1}^{\prime}, D_{21}\right]+\left[L_{2}^{\prime}, D_{11}\right]+\left[L_{3}^{\prime}, D_{21}\right]}
\end{aligned}
$$

Taking the modulus of the expectations of the both sides of Eq. (20) and applying the triangular inequality, we have

$$
\begin{aligned}
& \left|\left\langle\left[Z_{1}, L_{1}\right]\right\rangle\right| \\
& \quad \leq\left|\left\langle\left[L_{1}^{\prime}, D_{21}\right]\right\rangle\right|+\left|\left\langle\left[L_{2}^{\prime}, D_{11}\right]\right\rangle\right|+\left|\left\langle\left[L_{3}^{\prime}, D_{21}\right]\right\rangle\right| .
\end{aligned}
$$

By the uncertainty relation [13] and Eq. (17), we have

$$
\left|\left\langle\left[L_{k}^{\prime}, D_{i j}\right]\right\rangle\right| \leq 2 \Delta D_{i j} \Delta L_{k}^{\prime} \leq 2 \delta_{i j}(\psi) \Delta L_{k}^{\prime}
$$

Thus, we obtain the following consequence of the first noise commutation relation, Eq. (20),

$$
\begin{aligned}
\left|\left\langle\left[Z_{1}, L_{1}\right]\right\rangle\right| \leq & 2 \delta_{21}(\psi) \Delta L_{1}^{\prime}+2 \delta_{11}(\psi) \Delta L_{2}^{\prime} \\
& +2 \delta_{11}(\psi) \Delta L_{3}^{\prime} .
\end{aligned}
$$

Similarly, from the second noise commutation relation, Eq. (21), we obtain the following relation

$$
\begin{aligned}
\left|\left\langle\left[Z_{1}, L_{1}\right]\right\rangle\right| \leq & 2 \delta_{21}(\psi) \Delta L_{1}^{\prime}+2 \delta_{11}(\psi) \Delta L_{2}^{\prime} \\
& +2 \delta_{21}(\psi) \Delta L_{3}^{\prime} .
\end{aligned}
$$

Summing up both inequalities and using the relations $\Delta L_{1}^{\prime}, \Delta L_{2}^{\prime} \leq\left\|L_{1}\right\|=\left\|L_{2}\right\|$, we have

$$
\left|\left\langle\left[Z_{1}, L_{1}\right]\right\rangle\right| \leq\left(\delta_{11}(\psi)+\delta_{21}(\psi)\right)\left(2\left\|L_{1}\right\|+\Delta L_{3}^{\prime}\right) .
$$

By the inequality $(x+y)^{2} / 2 \leq x^{2}+y^{2}$, we have the lower bound of the imperfection

$$
\frac{\left|\left\langle\left[Z_{1}, L_{1}\right]\right\rangle\right|^{2}}{2\left(2\left\|L_{1}\right\|+\Delta L_{3}^{\prime}\right)^{2}} \leq \delta_{11}(\psi)^{2}+\delta_{21}(\psi)^{2}
$$

Let us consider the computational basis defined by the spin component of the $z$ direction and the angular momentum conservation law for the $x$ direction. Thus, we assume $L_{i}=X_{i}$ for $i=1,2$, so that

$$
\left\|L_{1}\right\|=\left\|L_{2}\right\|=1
$$

and that $L_{3}$ is considered as the $x$-component of the total angular momentum divided by $\hbar / 2$ of the ancilla system A. In order to maximize the bound in Eq. (26), suppose that the input state $|\psi\rangle$ is the spin state of the $y$ direction, i.e., $|\psi\rangle=\frac{1}{\sqrt{2}}(|0\rangle+|1\rangle)$. Then, by straightforward calculations we have

$$
\begin{aligned}
\delta_{11}(\psi)^{2}= & 2 \|\left|E_{00}^{10}\right\rangle\left\|^{2}+2\right\|\left|E_{01}^{10}\right\rangle\left\|^{2}+2\right\|\left|E_{10}^{00}\right\rangle \|^{2} \\
& +2 \|\left|E_{11}^{00}\right\rangle \|^{2}, \\
\delta_{21}(\psi)^{2}= & 2 \|\left|E_{00}^{10}\right\rangle\left\|^{2}+2\right\|\left|E_{01}^{00}\right\rangle\left\|^{2}+2\right\|\left|E_{10}^{10}\right\rangle \|^{2} \\
& +2 \|\left|E_{11}^{00}\right\rangle \|^{2} .
\end{aligned}
$$

Since $\sum_{c, d=0}^{1} \|\left|E_{c d}^{a b}\right\rangle \|^{2}=1$ for $a, b=0,1$, from Eq. (13) we have

$$
\begin{aligned}
\delta_{11}(\psi)^{2} & +\delta_{21}(\psi)^{2} \\
& \leq 4\left[1-F(00)^{2}\right]+4\left[1-F(10)^{2}\right] \\
& \leq 8\left[1-F\left(\mathcal{E}_{\alpha}, U_{\mathrm{CN}}\right)^{2}\right] .
\end{aligned}
$$

Since $\left[Z_{1}, L_{1}\right]=\left[Z_{1}, X_{1}\right]=2 i Y_{1}$, we have

$$
\left|\left\langle\left[Z_{1}, L_{1}\right]\right\rangle\right|=2 .
$$

Thus, from Eqs. (26), (27), (30), and (31), we have the following fundamental lower bound of the gate error probability

$$
\frac{1}{4\left(2+\Delta L_{3}^{\prime}\right)^{2}} \leq 1-F\left(\mathcal{E}_{\alpha}, U_{\mathrm{CN}}\right)^{2} \leq D_{C B}\left(\mathcal{E}_{\alpha}, U_{\mathrm{CN}}\right)
$$

In the following, we shall interpret the above relation in terms of the notion of the size of implementations for fermionic and bosonic ancillae separately.

We now assume that the ancilla A comprises qubits. Then, the size $s(\alpha)$ of the implementation $\alpha$ is defined to be the total number $n$ of the qubits included in $\mathbf{C}+\mathbf{T}+\mathbf{A}$. Then, we have 


$$
\Delta L_{3}^{\prime} \leq\left\|L_{3}\right\|=n-2 .
$$

Thus, we have the following lower bound of the gate error probability

$$
\frac{1}{4 s(\alpha)^{2}} \leq 1-F\left(\mathcal{E}_{\alpha}, U_{\mathrm{CN}}\right)^{2} \leq D_{C B}\left(\mathcal{E}_{\alpha}, U_{\mathrm{CN}}\right),
$$

with $s(\alpha)=n$. Therefore, it has been proven that if the computational basis is represented by the $z$-component of spin, any implementation with size $n$ which preserves the $x$-component of angular momentum cannot simulate the controlled-NOT gate within the error probability $1 /\left(4 n^{2}\right)$. In particular, any implementation on $\mathbf{C}+\mathbf{T}$ cannot simulate $U_{\mathrm{CN}}$ within the error probability $1 / 16$.

In current proposals [2,3], the external electromagnetic field prepared by the laser beam is considered to be a feasible candidate for the ancilla $\mathbf{A}$ to be coupled with the computational qubits $\mathbf{C}+\mathbf{T}$ via the dipole interaction. In this case, an analogous limit for bosonic ancillae is obtained by defining the size of the ancilla as 2 times the square root of the average number of photons, and thus the lower boud is inversely proportional to the average number of phtons. In fact, the ancilla state $|\xi\rangle$ is considered to be a coherent state, for which we have $(\Delta N)^{2}=\langle\xi|N| \xi\rangle=\langle N\rangle$, where $N$ is the number operator. We assume that the beam propagates to the $x$-direction with RHC polarization. Then, we have $L_{3}=2 N$, and hence

$$
\Delta L_{3}^{\prime}=2 \Delta N^{\prime}=2\left\langle N^{\prime}\right\rangle^{1 / 2} \leq 2(\langle N\rangle+2)^{1 / 2} .
$$

Thus, Eq. (34) holds with defining the size of implementation $\alpha$ by $s(\alpha)=2\langle N\rangle^{1 / 2}$ appropriately for the strong field, and hence Eq. (34) turns to be the relation

$$
\frac{1}{16\langle N\rangle} \leq 1-F\left(\mathcal{E}_{\alpha}, U_{\mathrm{CN}}\right)^{2} \leq D_{C B}\left(\mathcal{E}_{\alpha}, U_{\mathrm{CN}}\right)
$$

Formula (34) holds, therefore, appropriately for both fermionic and bosonic ancillae. In the most general case, Eq. (34) holds with $s(\alpha)=2+\Delta L_{3}^{\prime}$ dependent on the ancilla state, or with $s(\alpha)=2+\left\|L_{3}\right\|$ independent of the ancilla state.

The above limit on implementations of elementary gates cannot be circumvented by any choices of the set of universal gates. In fact, we can generally prove that in any set of universal gates, for any size limit s there is at least one gate which cannot be implemented within the error probability $1 /\left(k s^{2}\right)$ for some constant $k$. A proof runs as follows. Suppose that $U_{\mathrm{CN}}$ can be constructed from $m$ elementary gates. Let $U_{\mathrm{CN}}=U_{m} \cdots U_{1}$ and $\mathcal{E}_{\alpha}=\mathcal{E}_{m} \cdots \mathcal{E}_{1}$, where $\mathcal{E}_{i}$ is the operation of the best implementation of gate $U_{i}$ with size $s$. Then, $s(\alpha) \leq m s$, and hence from the chain property of $\mathrm{CB}$ distance [2,12], we have

$$
\frac{1}{4(m s)^{2}} \leq D_{C B}\left(\mathcal{E}_{\alpha}, U_{\mathrm{CN}}\right) \leq \sum_{i=1}^{m} D_{C B}\left(\mathcal{E}_{i}, U_{i}\right)
$$

Thus, one of $U_{i}$ must satisfy $1 /\left(4 m^{3} s^{2}\right) \leq D_{C B}\left(\mathcal{E}_{i}, U_{i}\right)$.

By modifying the model of a measurement due to Araki and Yanase [7], it can be shown that there is a physical implementation $\alpha$ of $U_{\mathrm{CN}}$ with any size $n$ satisfying the angular momentum conservation law such that $1-F\left(\mathcal{E}_{\alpha}, U_{\mathrm{CN}}\right)^{2}=O(1 / n)$. Thus, it is really possible to make the error probability small by making the ancilla large. The detailed construction will be discussed elsewhere.

Although it is difficult to envisage what the hardware of the quantum computer will be like, in order to realize a mobile quantum computer a fermionic ancilla appears to be plausible. The current theory demands the "threshold" error probability $10^{-5}-10^{-6}$ for each quantum gate [2, p. 482]. Thus, a single controlled-NOT gate would not in reality a unitary operation on a 2-qubit system but will be a unitary operation on a system with at least 100 qubits, as long as the computational basis is chosen as a spin component. The present investigation suggests that the current choice of the computational basis should be modified so that the computational basis commutes with the conserved quantity. Since the additive conserved quantity has degenerate spectrum on the multiple qubits, we may find such a computational basis comprised of orthogonal entangled states over a multiple-qubit system. Accordingly, the theory of fault-tolerant quantum computing based on single qubit errors should be modified to incorporate with such choice of the computational basis.

\section{ACKNOWLEDGMENTS}

The author thanks Julio Gea-Banacloche and Horace Yuen for helpful comments. This work was supported by the R\&D on Quantum Commun. Tech. Program of MPHPT, by the CREST project of the JST, and by the Grant-in-Aid for Scientific Research of the JSPS.

[1] P. W. Shor, SIAM J. Comput. 26, 1484 (1997).

[2] M. A. Nielsen and I . L. Chuang, Quantum Computation and Quantum Information (Cambridge University Press, Cambridge, 2000).

[3] See also, references in Ref. [2].

[4] S. Lloyd, Nature (London), 406, 1047 (2000).

[5] Y. J. Ng, Phys. Rev. Lett. 86, 2946 (2001).

[6] E. P. Wigner, Z. Physik 133, 101 (1952).

[7] H. Araki and M. M. Yanase, Phys. Rev. 120, 622 (1960).

[8] We denote the operator extended to a larger system by the same symbol as the original, e.g., $L_{1}$ instead of $L_{1} \otimes 1$.

[9] H. Stein and A. Shimony, in Foundations of Quantum Mechanics, edited by D'Espagnat (Academic, New York, 
1971); T. Ohira and P. Pearle, Am. J. Phys. 56, 692 (1988).

[10] This is the completely bounded norm 11] of the difference of two operations. It is equal to the diamond metric in $[12]$.

[11] V. I. Paulsen, Completely bounded maps and dilations, (Longman, New York, 1986).

[12] D. Aharonov, A. Kitaev, and N. Nisan, arXive e-print quant-ph/9806029 (1998) [in STOC 1997, 20-30].

[13] H. P. Robertson, Phys. Rev. 34, 163 (1929). 